\title{
New results from LOFAR
}

\author{
Vladislav Kondratiev ${ }^{1}$, \\ on behalf of Ben Stappers ${ }^{2}$ and the LOFAR Pulsar Working Group \\ ${ }^{1}$ ASTRON, the Netherlands Institute for Radio Astronomy, \\ Postbus 2, 7990 AA Dwingeloo, The Netherlands \\ email: vlad.kondratiev@gmail.com \\ ${ }^{2}$ Jodrell Bank Center for Astrophysics, School of Physics and Astronomy, \\ The University of Manchester, Manchester M13 9PL, UK \\ email: Ben.Stappers@manchester .ac.uk
}

\begin{abstract}
The LOw Frequency Array, LOFAR, is a next generation radio telescope with its core in the Netherlands and elements distributed throughout Europe. It has exceptional collecting area and wide bandwidths at frequencies from $10 \mathrm{MHz}$ up to $250 \mathrm{MHz}$. It is in exactly this frequency range where pulsars are brightest and also where they exhibit rapid changes in their emission profiles. Although LOFAR is still in the commissioning phase it is already collecting data of high quality. I will present highlights from our commissioning observations which will include: unique constraints on the site of pulsar emission, individual pulse studies, observations of millisecond pulsars, using pulsars to constrain the properties of the magneto-ionic medium and pilot pulsars surveys. I will also discuss future science projects and advances in the observing capabilities.
\end{abstract}

Keywords. pulsars: general, telescopes: LOFAR

\section{Introduction}

LOFAR is an interferometric array of dipole antenna stations distributed over the Netherlands and a few countries in Europe, that operates at the very low radio frequencies from 10 to $250 \mathrm{MHz}$. It consists of 24 core stations with the central part of about $300 \mathrm{~m}$ across occupied by 6 stations, which is called "Superterp". The Superterp provides a collecting area comparable to that of the 100-m Green Bank Telescope with a beam size of $\sim 0.5^{\circ}$ at $140 \mathrm{MHz}$. For the full core with a size of about $2 \mathrm{~km}$ across, it is already Arecibo-like collecting area and beam size of $\sim 5^{\prime}$. At the moment there are also 9 remote stations and 8 international stations included in the array. The latter are twice as big as Dutch stations and they can operate independently from the rest of the array. They are very powerful telescopes in their own right, each with collecting area comparable to that of the 64-m Parkes radio telescope.

LOFAR's frequency range spans from 10 to $250 \mathrm{MHz}$ which is achieved by using two types of dipoles, low-band antennas (LBA) at 10-90 MHz and high-band antennas (HBA) at 110-250 MHz. The HBA dipoles have bow-tie shape and grouped into tiles of 16 dipoles each. There are 48 tiles in Dutch stations and 96 in the international stations. LOFAR operates at the lowest radio frequencies visible from the Earth as at $10 \mathrm{MHz}$ there is a cut-off due to ionospheric reflection. Operating at these low frequencies, LOFAR covers the lowest 4 octaves of the radio window, that makes it a very unique telescope, the only one working at such low radio frequencies with huge instantaneous fractional bandwidth. 


\section{Cumulative S/N of PSR B2217+47 in 127 Simultaneous Tied-Array Beams}

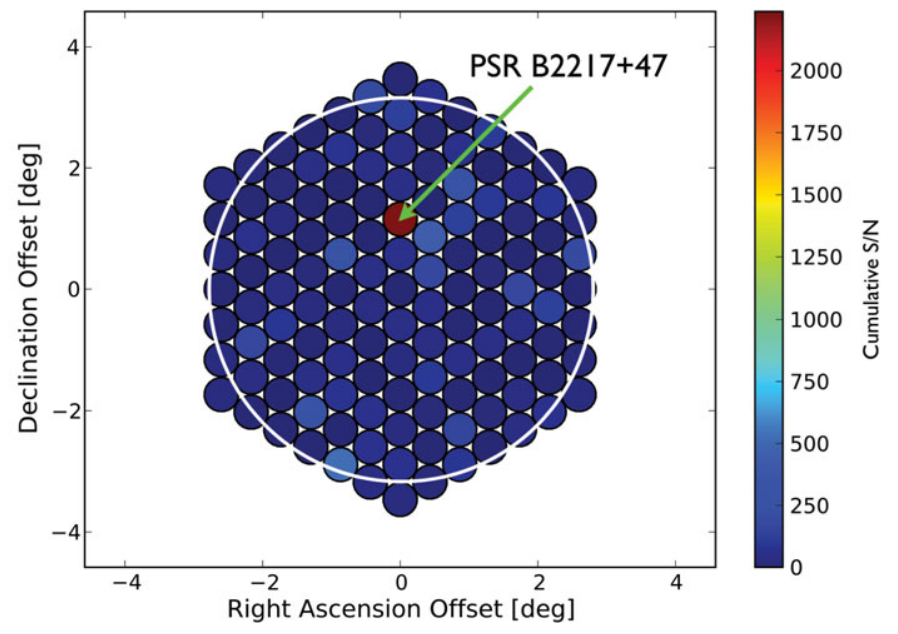

Figure 1. Hexagonal pattern of 127 tied-array beams formed around the pulsar B2217+47 using 12 HBA sub-stations of the Superterp. The beam with the pulsar is marked by the arrow. The cumulative signal-to-noise ratio is designated by the color scale. The white circle of about 5 degrees across represents the whole station beam of the single HBA sub-station.

\section{LOFAR Capabilities}

All of the beam-formed modes, capabilities and many commensal results are described in detail in our first LOFAR paper (Stappers et al. 2011). Here we highlight a few of those, focusing on new commissioning advancements.

Multiple station beams. LOFAR is a very flexible, electronically steered aperture array. It is possible to form multiple station beams on the sky and observe several pulsars simultaneously (Hessels et al. 2010; Stappers et al. 2011). This technology will be crucial for the SKA. By trading off bandwidth for beams, we can have as a standard up to 8 widely separated field-of-views (FOVs) and optionally up to 244 .

Tied-array beams. Within each of the station beams we can also form multiple tiedarray (TA) beams, applying proper phase delays between stations while adding them coherently. At the moment we can form TA beams only using 12 Superterp sub-stations, as they share the single clock. Figure 1 shows the hexagonal pattern of 127 TA beams formed around the pulsar B2217+47. The signal-to-noise ratio of the other TA beams is about one order of magnitude smaller than the beam at the location of the pulsar. With 127 tied-array beams we can cover the whole station beam which is shown by the white circle. The FOV of the station beam is large enough to cover the entire Andromeda galaxy and then we can map it with TA beams in one single observation. Moreover, we can form a few station beams to cover a larger FOV and customize individual narrow TA beams pointing at different targets. The highest number of TA beams formed in the commissioning observations so far was 217 ( 8 rings plus a central beam).

The list and description of all beam-formed modes that are well-tested and currently available to the wider community can be found on the LOFAR web-pages $†$. There are many possible modes or configurations, and the system is very flexible, to match different

$\dagger$ http://astron.nl/radio-observatory/observing-capabilities/ depth-technical-information/major-observing-modes/beam-form 

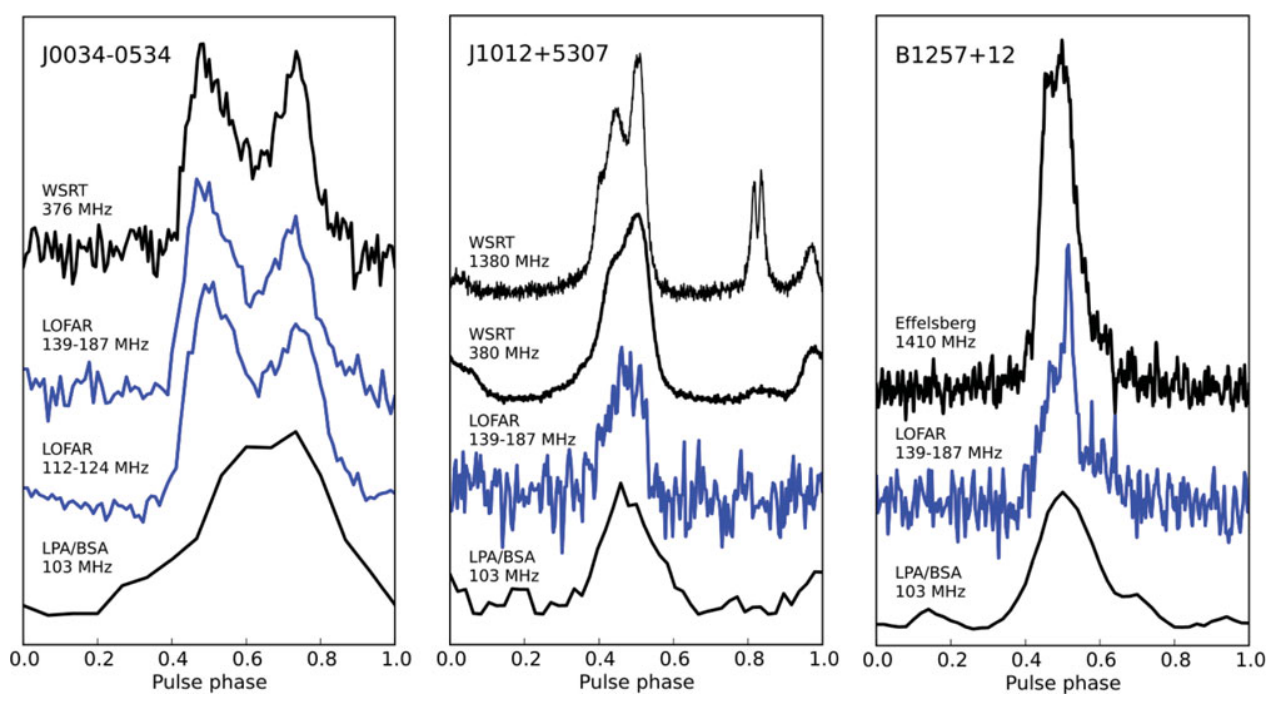

Figure 2. LOFAR profiles observed in the HBA bands (blue) for the three millisecond pulsars J0034-0534, J1012+5307, and B1257+12 in comparison with profiles at $103 \mathrm{MHz}$ with the BSA phased array in Puschino, and WSRT and Effelsberg profiles at higher frequencies (black). Each bin in the LOFAR profiles is about $20 \mu$ s wide. BSA profiles are from the EPN pulsar database (http://www.jb.man.ac.uk/research/pulsar/Resources/epn/browser.html).

science goals. Different data products can be recorded, namely total intensity, full Stokes parameters, or complex voltage data. All data are written in HDF5 $\ddagger$ format and we are already working to read it directly with DSPSR 9 (van Straten \& Bailes 2011) and PRESTO\| (Ransom 2001) pulsar software.

RFI. The RFI environment is very clean, much better than anticipated. The reasons for this is that a) we are using 12-bit ADCs at the station level, so the dynamic range is high; and b) the dipoles are located very low to the ground and do not pick up a much terrestrial interference. Typically, we flag about $1-2 \%$ of data in HBA, and $3-4 \%$ in LBA range. Below $30 \mathrm{MHz}$, however, the data get very contaminated by RFI.

Full-core single-clock. We are currently working on expanding the number of stations that use the single clock, from six stations on the Superterp to the whole core of 24 stations within $\sim 1 \mathrm{~km}$ radius. The work is ongoing and will be finished by the end of October 2012. This will further increase the raw sensitivity of the system by a factor 4!

\section{LOFAR Highlights}

Here I present some of our recent pulsar results; some are published or will be submitted soon.

Pulsar timing. LOFAR is very capable of, and we have already started doing, observations of millisecond pulsars (MSPs), as shown in Figure 2. LOFAR MSP profiles show a very high quality at such a low frequency in comparison with previously acquired data using the Puschino BSA phased-array at $103 \mathrm{MHz}$. These are the highestquality detections of these pulsars ever made below $200 \mathrm{MHz}$. For the MSP J0034-0534

\footnotetext{
$\ddagger$ Hierarchical Data Format 5, http://www.hdfgroup.org/HDF5/

I http://dspsr.sourceforge.net

|| http://www.cv.nrao.edu/ ${ }^{\text {sransom/presto/ }}$
} 


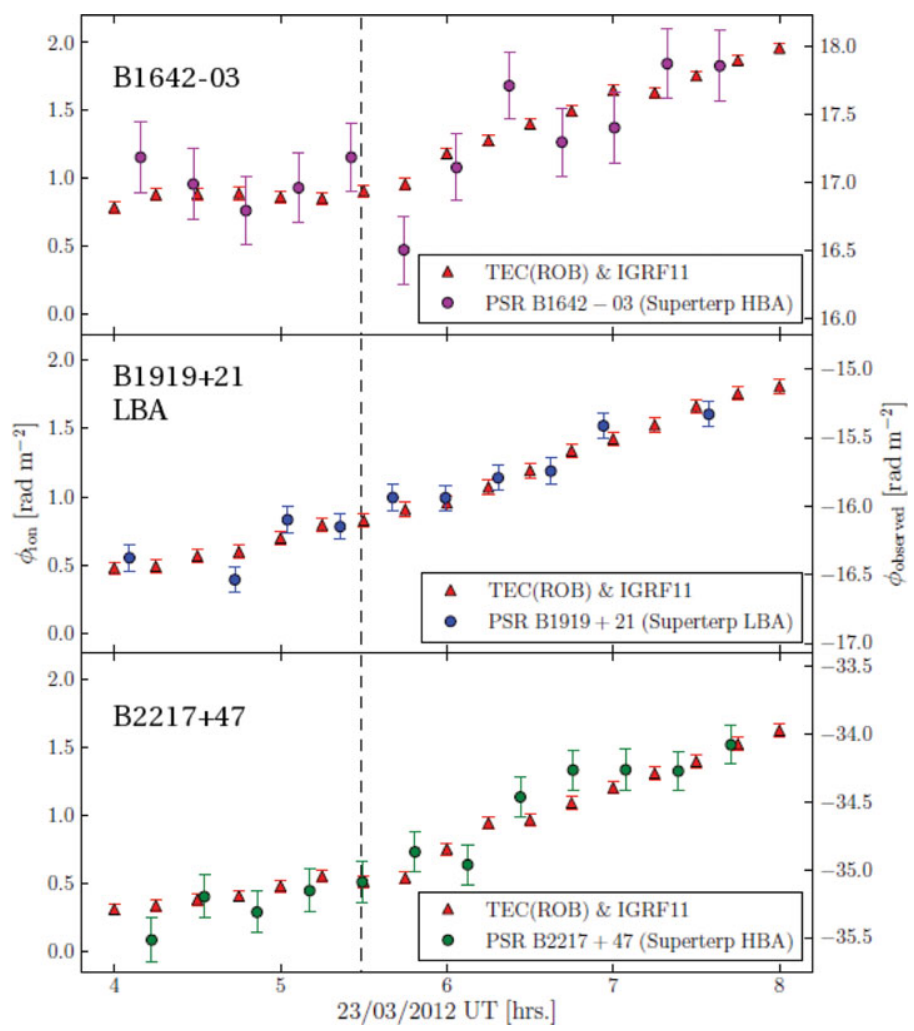

Figure 3. Observed Faraday depths, $\phi_{\text {observed }}$ (right axis), along the line-of-sights toward the pulsars B1642-03 (top), B1919+21 (middle), and B2217+47 (bottom) vs. observing time. Vertical dashed line designates the sunrise. The model output for each line-of-sight (red triangles) is shown on the left axis (Figure taken from Sotomayor-Beltran et al. 2012).

comparison of LOFAR profiles with the $376-\mathrm{MHz}$ WSRT profile shows that a small scattering tail becomes visible at lower frequencies (more apparent at 112-124 MHz), along with a slight change in the relative amplitudes of the two profile components. We have already started timing observations of MSPs to test the system and pipeline, and with the full-core single-clock we will start the real campaign of timing MSPs. LOFAR pulsar timing observations will be very important to get a handle on dispersion measure and pulse profile evolution crucial for high-precision timing at high radio frequencies.

Ionospheric Faraday rotation calibration. We started Faraday rotation monitoring to be able to measure accurately pulsar rotation measures (RMs). Figure 3 (Sotomayor-Beltran et al. 2012) presents the observed Faraday depths for three pulsars together with the model predictions based on the total electron content (TEC) maps from the Royal Observatory of Belgium (ROB) and the International Geomagnetic Reference Field (IGRF11). It can be clearly seen that our measurements (circles) match the model (red triangles) very well. We are now getting down to very robust and precise $\mathrm{RM}$ measurements of about $0.1 \mathrm{rad} \mathrm{m} \mathrm{m}^{-2}$. The observations presented used only $1 / 6$ of the LOFAR's available bandwidth, thus showing a great potential for even better RM measurements using the full bandwidth especially in the LBA band.

Dispersion measure vs. Profile variations. Hassall et al. (2012) studied dispersion measure (DM) and profile variations for four pulsars, B0329+54, B0809+74, B1133+16, 

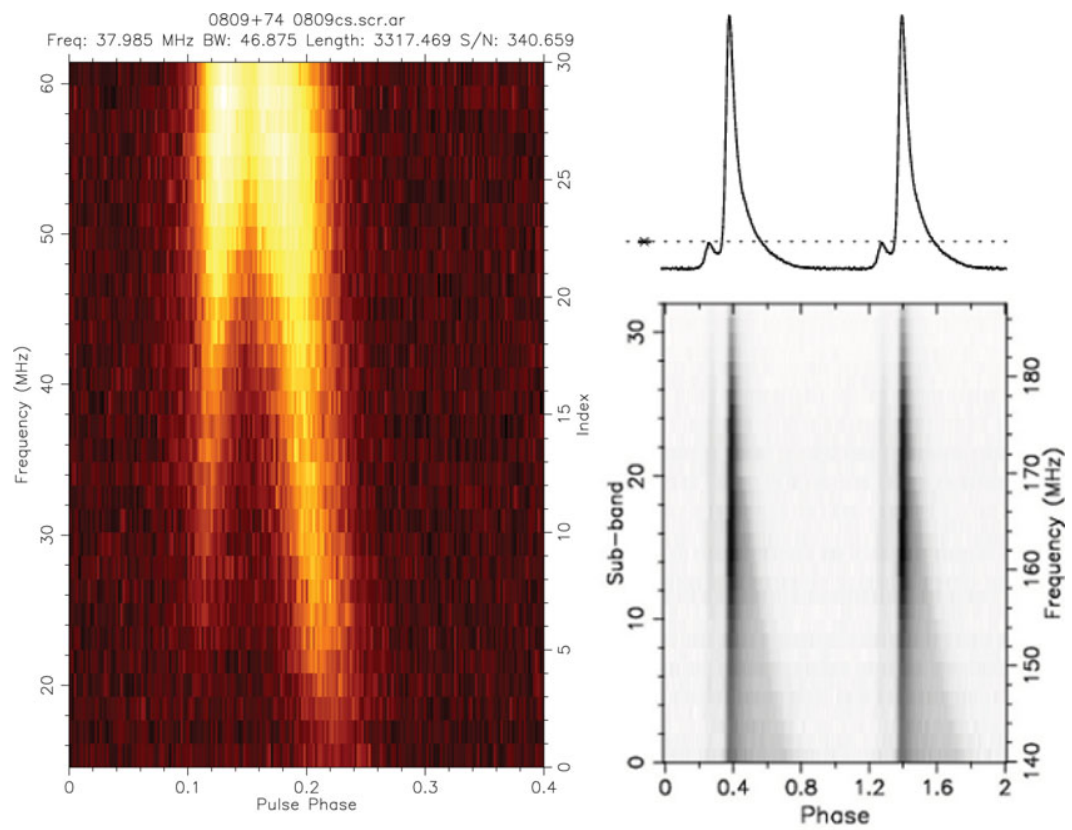

Figure 4. Left: LOFAR pulse average spectrum of PSR B0809+74 at frequencies $15-62 \mathrm{MHz}$. Right: Frequency-phase plot from one of the LOFAR HBA observations of PSR B2111+46 with the average pulse profile on the top. Grey scale designates the signal-to-noise ratio and remarkable evolution of the scattering tail is clearly visible.

and B1919+21 using simultaneous wideband observations with the LOFAR LBA and HBA at 40-190 MHz, the Lovell telescope at $1.4 \mathrm{GHz}$, and Effelsberg radio telescope at $8 \mathrm{GHz}$. We found that the dispersion law is correct to better than 1 part in $10^{5}$ across our observing band. We also put unique constraints on emission heights for these pulsars using aberration/retardation arguments and show that, for instance, in the case of the pulsar B1133+16 all radio emission comes from a small region less than $59 \mathrm{~km}$ in altitude at a height of less than $110 \mathrm{~km}$ above the neutron star surface (only $0.2 \%$ of the light cylinder). We found no evidence for the super-dispersion delay previously reported at low frequencies (Shitov \& Malofeev 1985; Kuzmin 1986) and suggest it could be caused by pulse profile evolution or a wrong fiducial point. We show that profile evolution has a siginificant impact on high-precision pulsar timing and should be taken into account.

Low-frequency single-pulse studies. Figure 4 (left) shows the remarkable profile evolution of the pulsar B0809+74 from 62 down to $15 \mathrm{MHz}$. We performed a thorough single-pulse analysis for the pulsars B0809+74 and B1133+16 that show quite interesting results. For more details about single-pulse studies of the pulsar B0809+74 see Kondratiev et al. (2012) in these proceedings.

Pilot pulsar surveys. We have already finished two pilot pulsar surveys with LOFAR. For more details about the survey setup, search pipelines and results, see Coenen et al. (2012) in these proceedings.

Low-frequency pulsar profiles. Some of the examples of LOFAR pulsar profiles at HBA and LBA bands were already shown in Stappers et al. (2011b). Currently we have already detected more than 110 pulsars in the HBA and 12 pulsars in the LBA. We expect these numbers to significantly increase in the very near future with the full-core single-clock, when the LOFAR raw sensitivity will be quadrupled. We are working on the 
ultimate LOFAR pulsar profile paper, and in particular on profile alignment with the high-frequency WSRT and Jodrell Bank data.

Scattering studies. LOFAR's low-frequency range and huge fractional bandwidth is ideal for pulsar scattering studies. Figure 4 (right) shows the benefits of the LOFAR's huge fractional bandwidth where you can see a remarkable scattering tail from the pulsar B2111+46 changing across the band. This allows us to study precisely the frequency dependency of scattering parameters of this and other pulsars.

\section{Future advancements}

LOFAR commissioning work is continuing and there are significant improvements which are coming by the end of Fall 2012, namely:

- Expanding single clock to the full LOFAR core (end of October 2012). This will quadruple LOFAR's raw sensitivity.

- Reading HDF5 data directly using DSPSR and PRESTO (nearly completed).

- Doubling (almost) of the available bandwidth to about $80 \mathrm{MHz}$ by implementing the 8-bit mode and potentially even 4-bit (end of October 2012).

- Implementing online RFI excision (removal from raw data).

- Creating sub-arrays and true Fly's Eye observations.

\section{Conclusions}

The results presented here have already proven the exceptional capabilities of the LOFAR and opened up the whole new window of comprehensive studies of pulsars at low frequencies. We have published first, intriguing results, with additional papers in preparation. The forthcoming implementation of the full-core single-clock, with the fourfold increase in sensitivity, will further enhance the LOFAR pulsar capabilities.

\section{Acknowledgements}

The LOFAR facilities in the Netherlands and other countries, under different ownership, are operated through the International LOFAR Telescope foundation (ILT) as an international observatory open to the global astronomical community under a joint scientific policy. In the Netherlands, LOFAR is funded through the BSIK program for interdisciplinary research and improvement of the knowledge infrastructure. Additional funding is provided through the European Regional Development Fund (EFRO) and the innovation program EZ/KOMPAS of the Collaboration of the Northern Provinces (SNN). ASTRON is part of the Netherlands Organization for Scientific Research (NWO).

\section{References}

Coenen, T., et al. 2012, these proceedings, arXiv:1211.1885

Hassall, T. E., Stappers, B. W., Hessels, J. W. T., et al. 2012, A\& A, 543, 66

Hessels, J. W. T., et al. 2010, ISKAF2010 Science Meeting, p. 25, arXiv:1009.1758

Kondratiev, V. I., et al. 2012, these proceedings, arXiv:1210.6994

Kuzmin, A. D. 1986, Soviet Astronomy Letters, 12, 325

Ransom, S. M. 2001, PhD thesis, Harvard University

Shitov, Y. P. \& Malofeev, V. M. 1985, Soviet Astronomy Letters, 11, 39

Sotomayor-Beltran, C., Sobey, C., et al. 2012, A\& $\&$, submitted

Stappers, B. W., Hessels, J. W. T., Alexov, A., et al. 2011, A $\& A$, 530, 80

Stappers, B., Hessels, J., Alexov, A., et al. 2011b, AIP Conf. Proc., 1357, 325

van Straten, W. \& Bailes, M. 2011, PASA, 28, 1 